\title{
Point cloud registration for agriculture and forestry crops based on calibration balls using Kinect V2
}

\author{
Sanzhang Zhou ${ }^{1,2}$, Feng Kang ${ }^{1,2^{*}}$, Wenbin $\mathrm{Li}^{1,2}$, Jiangming Kan ${ }^{1,2}$, Yongjun Zheng ${ }^{3}$ \\ (1. School of Technology, Beijing Forestry University, Beijing 100083, China; \\ 2. Key Lab of State Forestry and Grassland Administration on Forestry Equipment and Automation, \\ School of Technology, Beijing Forestry University, Beijing 100083, China; \\ 3. College of Engineering, China Agricultural University, Beijing 100083, China)
}

\begin{abstract}
For the process of point cloud registration, and the problem of inaccurate registration due to errors in correspondence between keypoints. In this paper, a registration method based on calibration balls was proposed, the trunk, branch, and crown were selected as experimental objects, and three calibration balls were randomly placed around the experimental objects to ensure different distances between two ball centers. Using the Kinect V2 depth camera to collect the point cloud of the experimental scene from four different viewpoints, the PassThrough filter algorithm was used for point cloud filtering in each view of the experimental scenes. The Euclidean cluster extraction algorithm was employed for point cloud clustering and segmentation to extract the experimental object and the calibration ball. The random sample consensus (RANSAC) algorithm was applied to fit the point cloud of a ball and calculate the coordinates of the ball center so that the distance between two ball centers under different viewpoints can be obtained by using the coordinates of the ball center. Comparing the distance between the ball centers from different viewpoints to determine the corresponding relationship between the ball centers from different viewpoints, and then using the singular value decomposition (SVD) method, the initial registration matrix was obtained. Finally, Iterative Closest Point (ICP) and its improved algorithm were used for accurate registration. The experimental results showed that the method of point cloud registration based on calibration balls can solve the problem of corresponding error of keypoints, and can register point clouds from different viewpoints of the same object. The registration method was evaluated by using the registration running time and the fitness score. The final registration running time of different experimental objects was not more than $6.5 \mathrm{~s}$. The minimum fitness score of the trunk was approximately 0.0001 , the minimum fitness score of the branch was approximately 0.0001 , and the minimum fitness score of the crown was approximately 0.0006 .
\end{abstract}

Keywords: point cloud registration, calibration balls, Kinect V2, ICP

DOI: $10.25165 /$ j.ijabe.20201301.5077

Citation: Zhou S Z, Kang F, Li W B, Kan J M, Zheng Y J. Point cloud registration for agriculture and forestry crops based on calibration balls using Kinect V2. Int J Agric \& Biol Eng, 2020; 13(1): 198-205.

\section{Introduction}

In recent years, plant phenotypic automated detection technology has played an important role in agricultural research and crop breeding ${ }^{[1]}$. The phenotype of plants is the result of the joint dynamics of genes and the environment ${ }^{[2]}$. Three-dimensional reconstruction techniques can be used to obtain phenotypic parameters of plants and guide agricultural production. The three-dimensional imaging system for plants can be mainly divided into stereo laser scanning technology, stereoscopic vision technology and structured light technology ${ }^{[3]}$. Colaço et al. ${ }^{[4]}$ used a two-dimensional laser and Global Navigation Satellite System (GNSS) receiver system to combine the convex-hull and the

\section{Received date: 2019-04-14 Accepted date: 2019-12-22}

Biographies: Sanzhang Zhou, Postgraduate, research interests: machine vision, Email: zhousanzhang@bjfu.edu.cn; Wenbin Li, PhD, Professor, research interests: forestry machine automation, Email: leewb@bjfu.edu.cn; Jiangming Kan, $\mathrm{PhD}$, Professor, research interests: computer vision, pattern recognition, Email: kanjm@bjfu.edu.cn; Yongjun Zheng, PhD, Professor, research interests: automation, aerial spraying, Email: zyj@cau.edu.cn

*Corresponding author: Feng Kang, PhD, Professor, research interests: agricultural and forestry automation, precision spraying. School of Technology, Beijing Forestry University, Beijing 100083, China. Tel: +86-15201430577, Email: kangfeng98@bjfu.edu.cn. alpha-shape algorithm to reconstruct the three-dimensional shape of citrus trees for improved management of agriculture. Li et al. ${ }^{[5]}$ employed a monocular stereo vision system combined with AD-Census and adaptive support-weight (ASW) algorithms to match images and reconstruct the three-dimensional shape of crops. Li et al. ${ }^{[6]}$ applied structured light technology to collect point cloud information of growing crops, the point cloud was segmented by markers to reconstruct the geometric three-dimensional shape of the crop, and successfully detected the sprouting and bifurcation of crops. At present, three-dimensional reconstruction of crops based on a three-dimensional point cloud is developing rapidly. Using a depth camera to collect point cloud information of crops can avoid the influence of natural light, effectively construct a three-dimensional model of crops, and obtain plant phenotypic data $^{[7]}$. A Kinect V2 depth camera was exploited for 3D scanning, this camera has the advantages of speed, low cost, accuracy, and convenience, and was widely used in robotic navigation and localization $^{[8]}$, somatosensory technology ${ }^{[9]}$, the medical field ${ }^{[10]}$, the education field ${ }^{[11]}$ and other fields, The scope of application has also been extended to the field of agricultural 3D reconstruction. Vázquez-Arellano et al. ${ }^{[12]}$ utilized Kinect V2 to map 3D images to color images for 3D reconstruction of maize plants. The registration operation was performed using the ICP algorithm, the ground point cloud was separated and the corn plants were 
extracted using the random sample consensus (RANSAC) algorithm. Gai et al. ${ }^{[13]}$ employed the Kinect V2 camera to capture and fuse two-dimensional images and point clouds of indoor and outdoor crops. The filter operation was performed by the cut-off filter, and the ground point cloud was separated by the RANSAC algorithm to reconstruct the three-dimensional model of the crop. Among these operations, point cloud registration is the key to $3 \mathrm{D}$ reconstruction ${ }^{[14]}$. Today, the technology of point cloud automatic registration is mainly to find local features of the point cloud, such as normal aligned radial feature (NARF) key points ${ }^{[15]}$, local surface patch (LSP) local feature ${ }^{[16]}$, and scale-invariant feature transform (SIFT) key points ${ }^{[17]}$, and then ICP and its improved algorithm are exploited for point cloud registration. However, due to the influence of the flying point, it is easy to form an incorrect correspondence relationship, resulting in the problem of incorrect registration. In this paper, the Kinect V2 depth camera was utilized to collect point cloud data. Considering the complexity of the forest environment, color information is not employed as additional information for point cloud registration. Three balls were placed around the experimental object, and point cloud registration was carried out according to the corresponding relationship of the same ball center under different viewpoints, this approach effectively avoided the wrong corresponding relationship. The registration method based on calibration balls is suitable for point cloud registration of forests, canopies and other crops in different environments, and has a wide range of applications.

\section{Materials and methods}

\subsection{Theoretical background}

The sketch of the experimental scene is shown in Figure 1. The experimental object was placed in the center and three calibration balls were placed around the experimental object. The placement of the calibration balls is arbitrary and the lengths of the calibration balls $\left(L_{1}, L_{2}, L_{3}\right)$ are different. Using the ball center as the key point from each viewpoint, the system was programmed according to the different distances of the ball center to determine the corresponding relationship. During the experiment, four viewpoints completely covering the experimental object were arbitrarily selected, and the visible portion of each ball was ensured to be greater than or equal to approximately $1 / 3$ of the whole body at each viewpoint. Taking viewpoint 1 and 2 as examples, the process of determining the corresponding ball center was analyzed in Figure 2. In viewpoint 1, the balls were randomly numbered, and the distances between any two balls were calculated as $d_{12}, d_{23}$, $d_{31}$ using the fitted ball coordinates. The same method was used in viewpoint 2 , and the calculated distance was defined as $d_{12}^{\prime}, d_{23}^{\prime}$, $d^{\prime}{ }_{31}$. Theoretically, the distance between two ball centers from different viewpoints should be equal. However, the value varied slightly due to the ball fitting error. A threshold $D_{t h}$ was used to check the correspondence, which was set to $0.02 \mathrm{~m}$. The same progress was also conducted in viewpoints 3 and 4 .

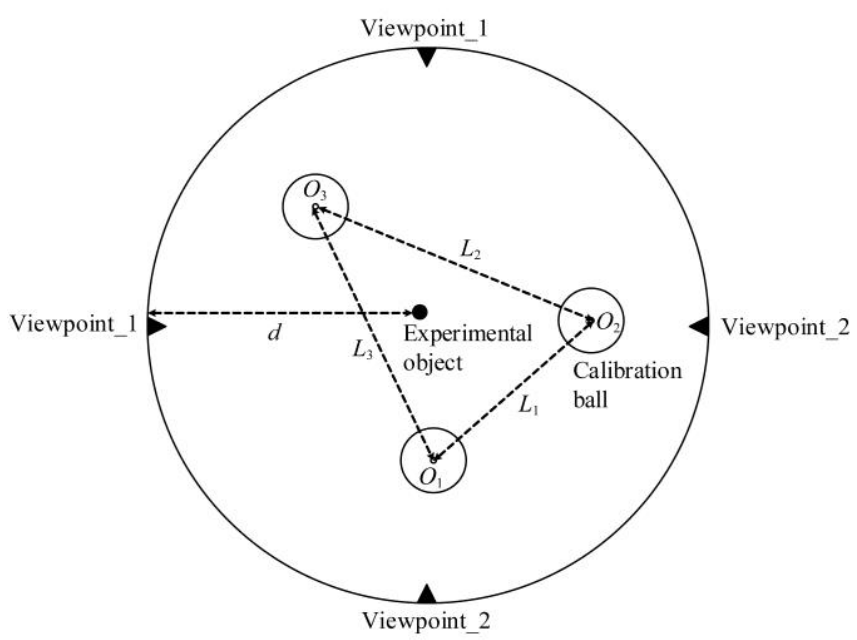

Figure 1 Draft of the experimental scene

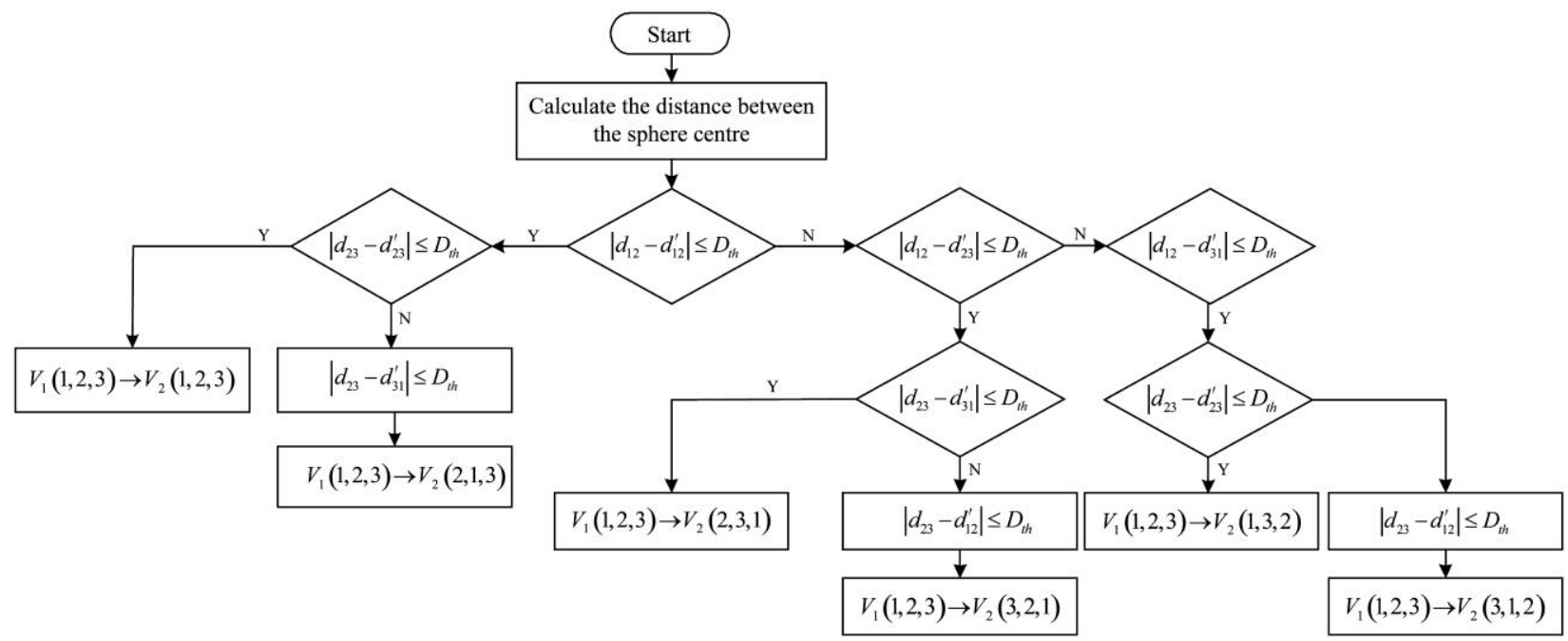

Figure 2 Analyze the corresponding ball center

The core issue of 3D multi-view point cloud registration is to find the transformation matrix between various viewpoints. The transformation matrix is composed of a $4 \times 4$ matrix shown as Equation (1).

$$
\text { transform }=\left[\begin{array}{cccc}
a_{11} & a_{12} & a_{13} & p_{x} \\
a_{21} & a_{22} & a_{23} & p_{y} \\
a_{31} & a_{32} & a_{33} & p_{z} \\
\boldsymbol{t}_{\boldsymbol{x}} & \boldsymbol{t}_{\boldsymbol{y}} & \boldsymbol{t}_{z} & \boldsymbol{s}
\end{array}\right]
$$

where, $\left(\begin{array}{lll}\boldsymbol{a}_{11} & \boldsymbol{a}_{12} & \boldsymbol{a}_{13} \\ \boldsymbol{a}_{21} & \boldsymbol{a}_{22} & \boldsymbol{a}_{23} \\ \boldsymbol{a}_{31} & \boldsymbol{a}_{32} & \boldsymbol{a}_{33}\end{array}\right)$ represents the rotation matrix $R,\left(P_{x}, P_{y}\right.$,

$\left.P_{z}\right)$ represents the translation matrix $t,\left(t_{x}, t_{y}, t_{z}\right)$ represents the perspective transformation part, and $s$ represents the scale factor of the transformation. The rotation matrix $R$ and translation matrix $t$ can be solved by using three pairs of corresponding points from different viewpoints when the perspective transformation and 
proportion are not involved in the registration of three-dimensional multi-view point clouds, the perspective transformation is 0 and the scale factor is 1 . Based on the principle of $\mathrm{SVD}^{[18]}$, the rotation matrix $R$ and the translation matrix $t$ are derived. Two corresponding point sets $p=\left\{p_{1}, p_{2} \ldots p_{n}\right\}$ and $q=\left\{q_{1}, q_{2} \ldots q_{n}\right\}$ are found in the $R^{d}$ dimension space. Assuming that the coordinates of the corresponding points in the source point cloud are $p=\left\{p_{1}\right.$, $\left.p_{2} \ldots p_{n}\right\}$ and the coordinates of the corresponding points in the target point cloud are $q=\left\{q_{1}, q_{2} \ldots q_{n}\right\}$, calculating the rigid transformation between the source point cloud and the target point cloud, that is, $R$ and $t$ can be transformed into Equation (2). $w_{i}>0$ represents the weight of each pair of corresponding points.

$$
(R, t)=\underset{R \in S O(d), t \in R^{d}}{\operatorname{argmin}} \sum_{i=1}^{n} w_{i}\left\|\left(R p_{i}+t\right)-q_{i}\right\|^{2}
$$

The weighted centroid and the center vector of the point set are calculated and are shown as Equations (3)-(5).

$$
\begin{gathered}
\bar{p}=\frac{\sum_{i=1}^{n} w_{i} p_{i}}{\sum_{i=1}^{n} w_{i}} \\
\bar{q}=\frac{\sum_{i=1}^{n} w_{i} q_{i}}{\sum_{i=1}^{n} w_{i}} \\
x_{i}:=p_{i}-{ }^{-} p \quad y:=q- \\
S=X W Y^{T}
\end{gathered}
$$

where, $\sum$ is a diagonal matrix composed of singular values; and $U$ and $V$ are diagonal matrices. $d \times d$ covariance matrix is calculated and is shown as Equation (6). $X, Y$ are matrix of $d \times n$ dimensions, which are columns of matrix $X, Y$, and $w$ represents the diagonal matrix, that is, $W=\operatorname{diag}\left(w_{1}, w_{2} \ldots w_{n}\right) . \quad s$ performs singular value decomposition and is shown as Equation (7).

$$
\boldsymbol{S}=\boldsymbol{U} \sum \boldsymbol{V}^{T}
$$

The rotation matrix $R$ and the translation matrix $t$ of the corresponding point pairs in the source point cloud and the target point cloud are calculated by Equations (8) and (9).

$$
\boldsymbol{R}=\boldsymbol{V}\left(\begin{array}{ccccc}
1 & & & & \\
& 1 & & & \\
& & \ddots & & \\
& & & 1 & \\
& & & & \operatorname{det}\left(\boldsymbol{V} \boldsymbol{U}^{T}\right)
\end{array}\right)
$$

\subsection{Experimental equipment}

The parameters of the Kinect V2 depth camera used in this paper are shown in Table $1^{[19]}$. The computer that processes the point cloud information uses Intel(R) Core(TM) i5-7500 CPU @ 3.40GHz, 8G RAM, Intel(R) HD Graphics 630 configuration.
Table 1 Kinect V2 relevant parameters

\begin{tabular}{lc}
\hline \multicolumn{1}{c}{ Feature } & Value \\
\hline Infrared (IR) camera resolution & $512 \times 424$ pixels \\
RGB camera resolution & $1920 \times 1080$ pixels \\
Field of view & $70 \times 60$ degrees \\
Frame rate & 30 frames per second \\
Operative measuring range & between $1.4 \mathrm{~mm}($ @ $0.5 \mathrm{~m}$ range $)$ \\
Object pixel size (GSD) & and $12 \mathrm{~mm}(@ 4.5 \mathrm{~m}$ range $)$ \\
\hline
\end{tabular}

The experiment exploited the software tool program development kit Kinect for Windows SDK provided by Microsoft Corporation and the application programming interface API. Based on the Point Cloud Library (PCL) library ${ }^{[15]}$, the point cloud acquisition program was used to collect the point cloud of the experimental object. According to the relevant literature, when the Kinect $\mathrm{V} 2$ is between $0.5-3 \mathrm{~m}$ away from the experimental object, the error of the horizontal and vertical viewing angles is less than $2 \mathrm{~mm}^{[20]}$. To ensure the accuracy of the Kinect V2, the experimentally set image acquisition distance $d$ is approximately $2.2 \mathrm{~m}$, as shown in Figure 1. The experimental objects used are a trunk, branches, and a crown, and the relevant parameters of the experimental objects are shown in Figure 3. The indoor and outdoor environment of the experiment is shown in Figure 4.

\subsection{Experimental method}

The flow chart of the registration is shown in Figure 5. The point cloud registration method based on the calibration ball was used to first remove the background noise by point cloud filtering and then performed the European clustering to extract the experimental object and remove the outliers. The point cloud registration method based on calibration balls was divided into two stages: coarse registration and fine registration. The point cloud coarse registration process is shown in Figure 6. And the aim was to obtain the initial registration matrix by using the corresponding relationship of the ball centers from different viewpoints. The point clouds collected at viewpoint 1-4 were considered as the first to fourth frame $\left(\mathrm{F}_{1}-\mathrm{F}_{4}\right)$, respectively. And the merged point clouds were defined as $M_{1}, M_{2}$, and $M_{3}$, respectively. $F_{1}$ was the target point cloud while $\mathrm{F}_{2}$ was the source point cloud. A merged point cloud $M_{1}$ can be obtained by registering $F_{2}$ to $F_{1}$, which would be considered as the target point cloud and $\mathrm{F}_{3}$ was employed as the source point cloud for the next registration. All the rest were determined in the same way. A complete 3D point cloud of the object $\left(\mathrm{M}_{3}\right)$ was established through the whole progress. Fine registration was a comparative analysis using ICP, Iterative Closest Point NonLinear (NL_ICP), and Iterative Closest Point with Normals (N_ICP).
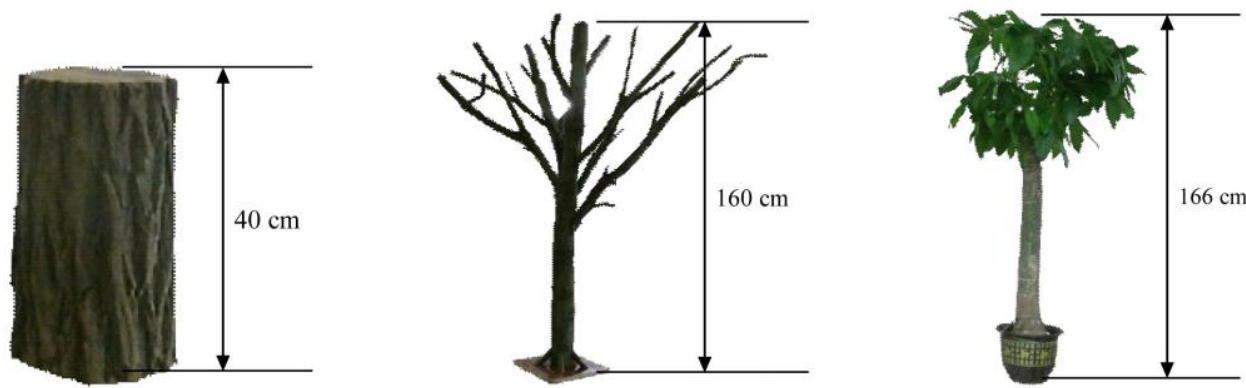

Figure 3 Experimental object 

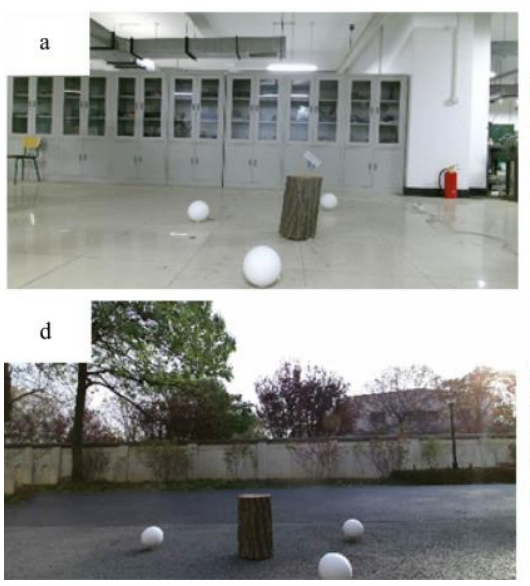

Note: $a, b, c$ are indoor scenes; $d, e, f$ are outdoor scenes.

Figure 4 Experimental scenes
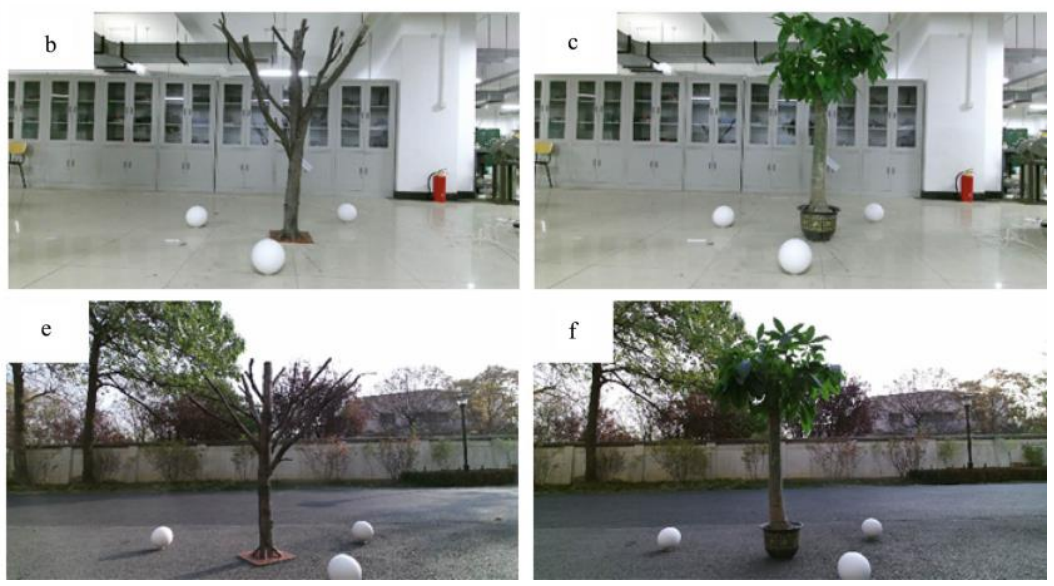

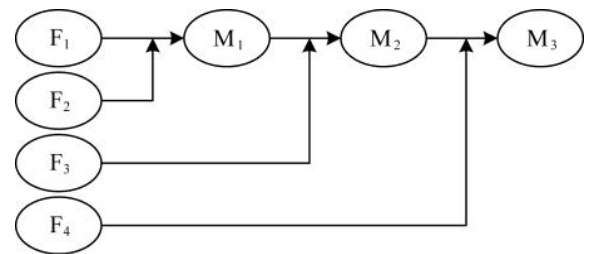

Figure 5 Point cloud processing

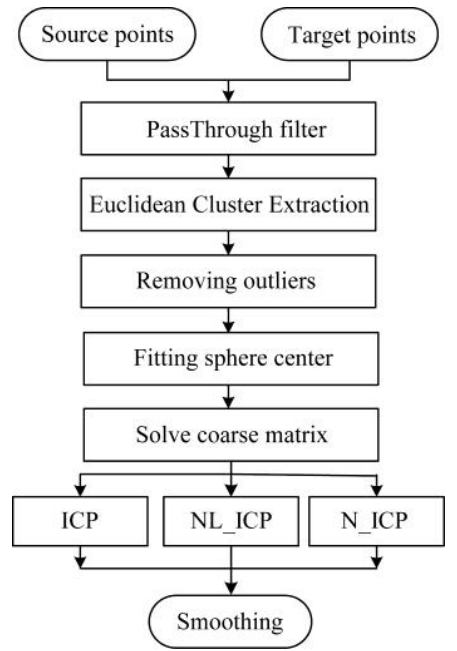

Figure 6 Point cloud coarse registration process

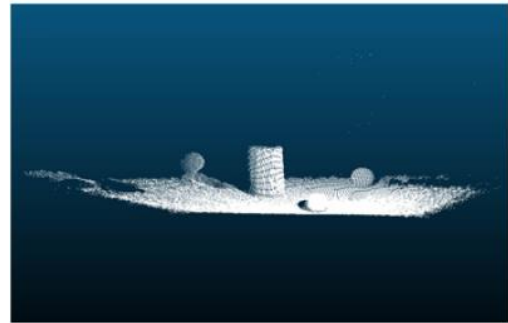

a. Trunk in an indoor experimental environment

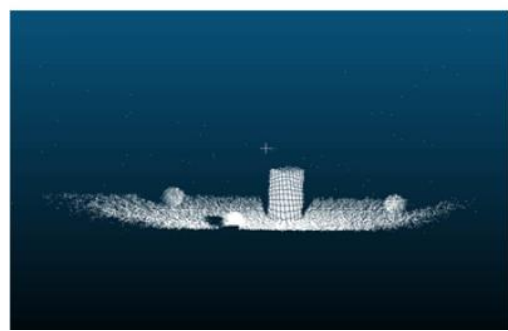

d. Trunk in an outdoor experimental environment

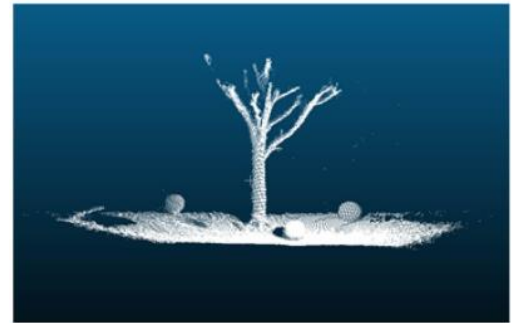

b. Branches in an indoor experimental environment

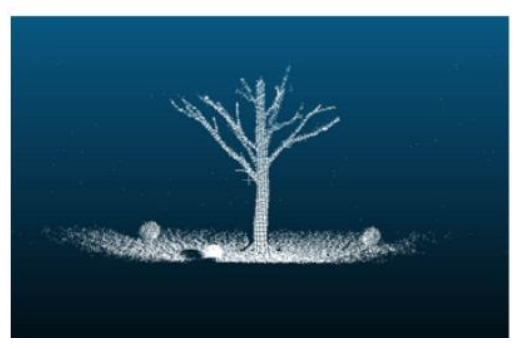

e. Branches in an outdoor experimental environment

Figure 7 Filtered point cloud

\subsection{Point cloud preprocessing}

The Kinect V2 depth camera has a large field of view, and the point cloud contains more noise. It is necessary to filter the point cloud after the acquisition. PassThrough filter ${ }^{[21]}$ is to remove noise points from the point cloud which are not within a specific range by setting the upper and lower limits. According to the coordinate system characteristics of the Kinect V2 depth camera and range of accuracy ${ }^{[20]}, 0.5$ and $2.5 \mathrm{~m}$ from the camera to the object were respectively set as the lower and upper distance limits for PassThrough filtering. The filtered point cloud is shown in Figure 7. From filtered point cloud, the ball, the experimental object and the ground point cloud are not separated, so the algorithm is needed for segmentation and clustering. In this paper, the European clustering ${ }^{[22]}$ based on the RANSAC principle was used to segment the ground, cluster the point clouds, and segment the experimental objects and three balls. At the same time, the outliers of the segmented objects were removed by the K-means method ${ }^{[23]}$, to avoid affecting the registration operation. When the registration method based on the calibration ball was performed, the initial registration matrix should be obtained by using the ball center. Therefore, the ball center should be fitted from different viewpoints, and the correlation coefficients such as the ball center and the radius of the ball can be obtained according to the random sampling consistency of RANSAC in PCL ${ }^{[24]}$. 


\subsection{Point cloud registration and point cloud smoothing}

Coarse and fine registrations were two parts of the point cloud registration method based on calibration balls. Coarse registration was to obtain the initial registration matrix from the SVD method according to the corresponding relationship of ball centers in different viewpoints. The ICP algorithm ${ }^{[25]}$ and its improved algorithm were employed for fine registration. The initial registration matrix is ensured that the point cloud has a good initial position $^{[26]}$, which is conducive to the fine registration of the point cloud. After registration, point clouds were smoothed using a smoothing algorithm based on the least-squares method ${ }^{[27]}$.

\section{Results and discussion}

The results of the indoor and outdoor trunk registration are shown in Figure 8. The number of point clouds in the registration process is shown in Figure 9a. The program running time is shown in Figure 9b. The fitness score is the sum of the squared distance of the source and target point clouds provided in PCL. The quality of the registration results by different methods of the same object can be judged according to the value of the corresponding fitness score. The smaller the score, the better the registration result. However, there is no comparability for the registration results of different experimental objects using their fitness scores ${ }^{[28]}$. The fitness score of the trunk is shown in Table 2. Figure 9a shows that the number of point clouds is increasing because of superimposed registration. At the same time, from Figure $9 \mathrm{~b}$, we can conclude that the registration time of each registration method is increasing because of the increase in the number of point clouds. To simplify the analysis process and evaluate the accuracy and time of model registration, the running time and fitness score of the fourth frame registration to the first, second and third frames were analyzed. The evaluation method of the branch and crown registration results was consistent with the trunk. In the indoor scenes, for the trunk, the final registration time can reach $0.429 \mathrm{~s}$, the corresponding method is the N_ICP method. The minimum fitness score of registration can reach 9.12E-05, and the corresponding registration method is NL_ICP. In the outdoor scene, for the trunk, the final registration time can be as short as $0.448 \mathrm{~s}$, and the corresponding registration method is N_ICP. The fitness score can be as small as 8.64E-05, and the corresponding registration method is NL_ICP. From the above trunk registration results, both indoor and outdoor conditions, the registration method N_ICP can achieve the shortest registration time, and the registration method NL_ICP can attain the minimum fitness score.
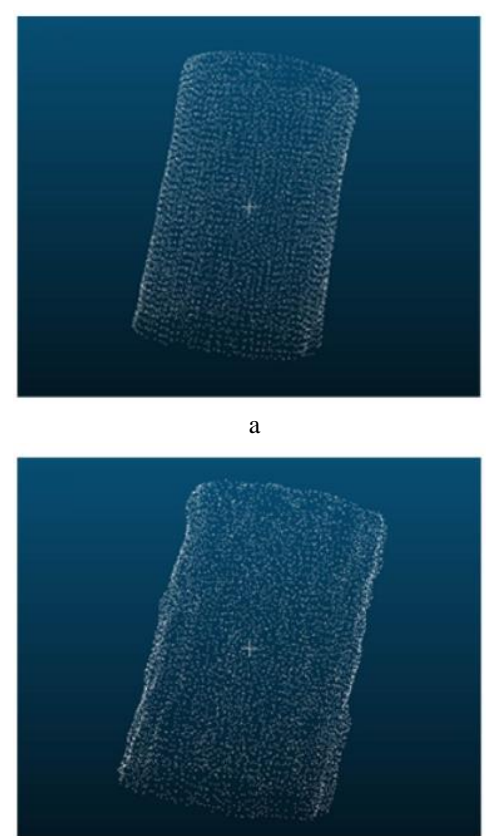

d

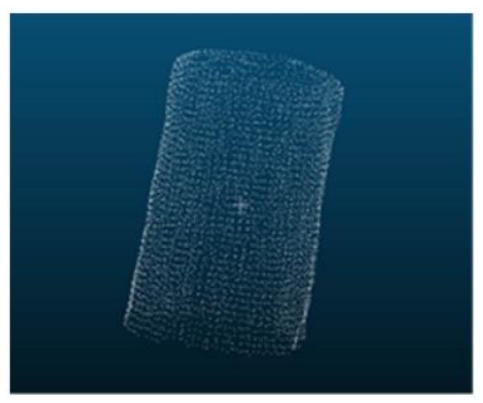

b

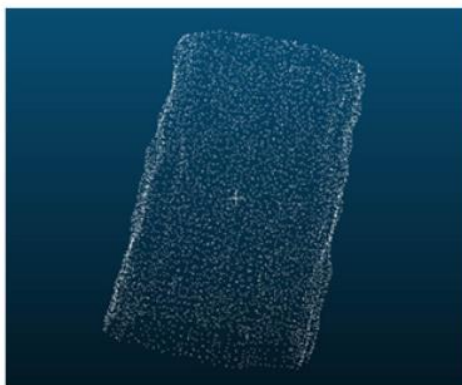

e

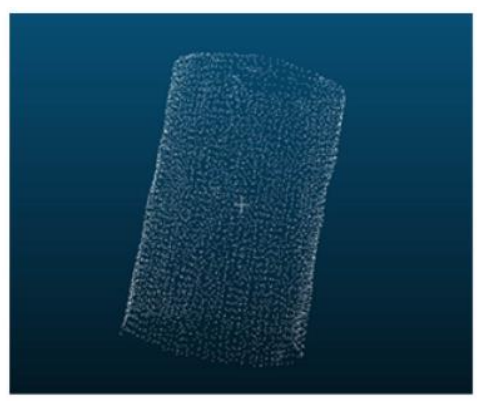

$\mathrm{c}$

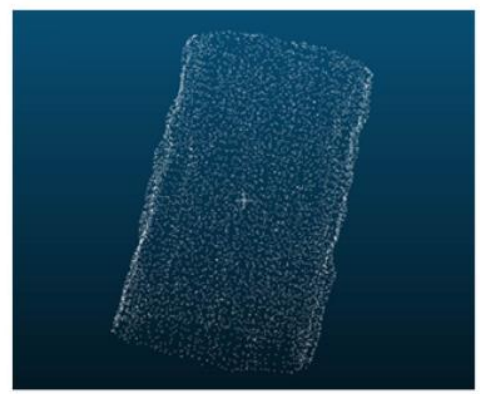

$\mathrm{f}$

Note: $a, b, c$ are in an indoor experimental environment; $d$, e, $\mathrm{f}$ are in an outdoor experimental environment; a, d registration using ICP method; $\mathrm{b}$, e registration using NL_ICP method; $\mathrm{c}$, f registration using N_ICP method.

Figure 8 3D model of the trunk

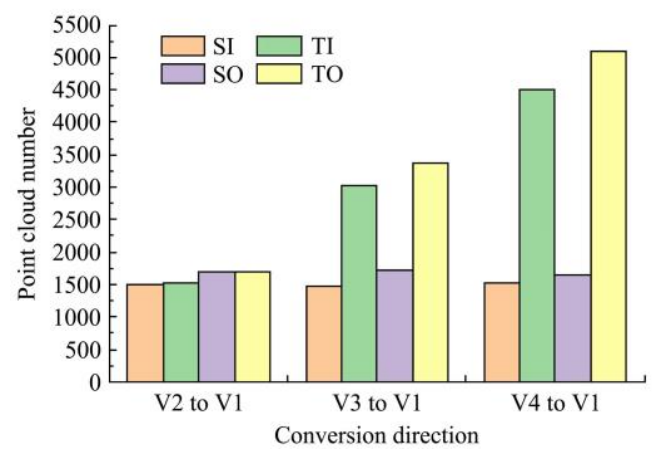

a. A trunk point cloud number variation

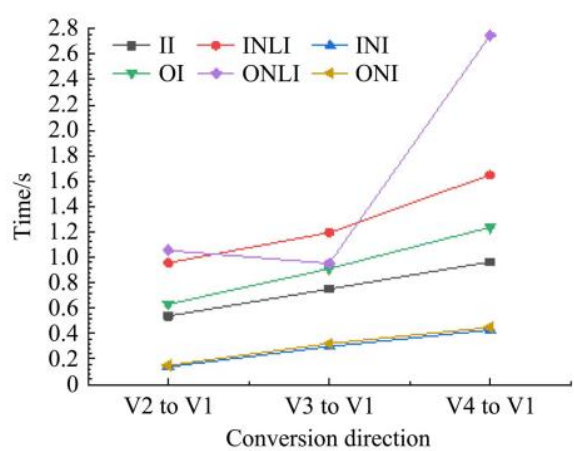

b. Running time of each step of various registration methods

Note: SI: source_indoor; TI: target_indoor; SO: source_outdoor; TO: target_outdoor; II: indoor_ICP; INLI: indoor_NL_ICP; INI: indoor_N_ICP; OI: outdoor_ICP; ONLI: outdoor_NL_ICP; ONI: outdoor_N_ICP. The same below.

Figure 9 Registration evaluation 
Table 2 Trunk fitness score

\begin{tabular}{cccc}
\hline \multirow{2}{*}{$\begin{array}{c}\text { Algorithms in different } \\
\text { environments }\end{array}$} & \multicolumn{3}{c}{ Conversion direction } \\
\cline { 2 - 4 } & V2 to V1 & V3 to V1 & V4 to V1 \\
\hline II & 0.005319 & 0.002181 & 0.000169 \\
OI & 0.002912 & 0.003947 & 0.000182 \\
INLI & 0.005833 & 0.001721 & $9.12 \mathrm{E}-05$ \\
ONLI & 0.003142 & 0.003155 & $8.64 \mathrm{E}-05$ \\
INI & 0.003767 & 0.002313 & 0.000223 \\
ONI & 0.002869 & 0.003386 & 0.000111 \\
\hline
\end{tabular}

The results of the branch registration indoors and outdoors are shown in Figure 10. The number change of points in point clouds in the registration process is shown in Figure 11a, the running time of the program is shown in Figure 11b, and the fitness score of tree registration is shown in Table 3. Figures $11 \mathrm{a}$ and $11 \mathrm{~b}$, show that the registration time of point clouds increases with the increase of the number of points in the point clouds in the registration process. In indoor scenes, for the branch, the final registration time can reach $1.125 \mathrm{~s}$, the corresponding registration method is N_ICP. The minimum fitness score can reach 0.001956, and the corresponding registration method is NL_ICP. In the outdoor scene, for the branch, the final registration time can be as short as $1.375 \mathrm{~s}$, the corresponding registration method is N_ICP, and the fitness score of the three registration methods is 0.000153 , 0.000149 , and 0.000143 , respectively. There is no big difference in the value. From the above experimental results, both indoor and outdoor environments, N_ICP achieved the shortest registration time, and NL_ICP performed a good fitness score in indoor and outdoor environments, with a wide range of applications.

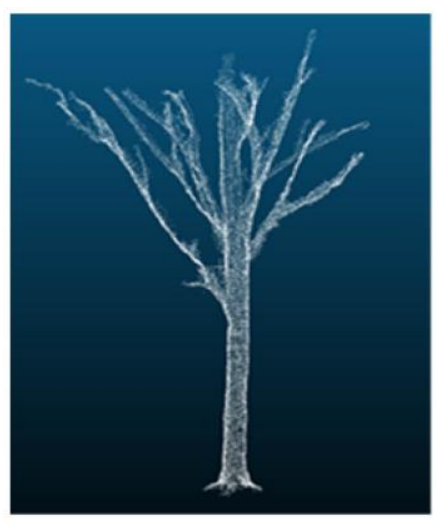

a

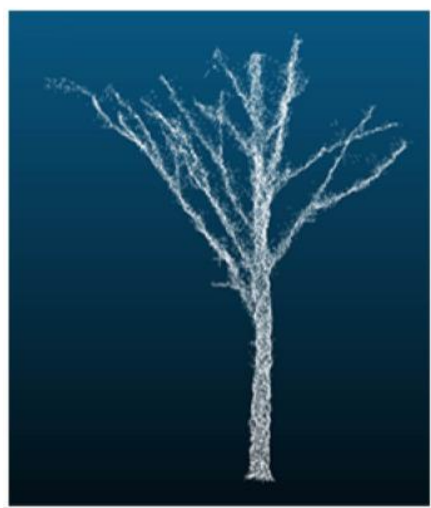

d

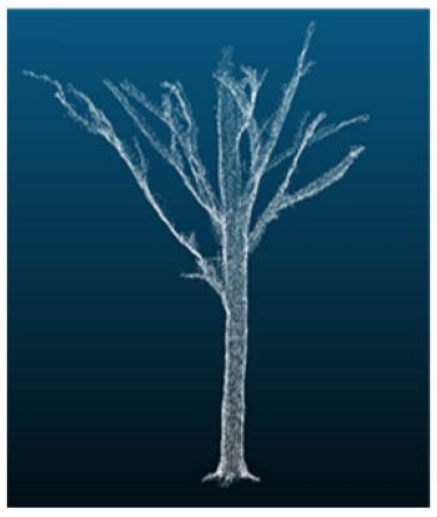

b

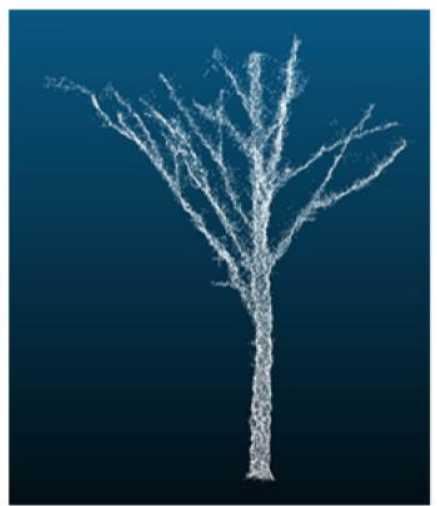

e

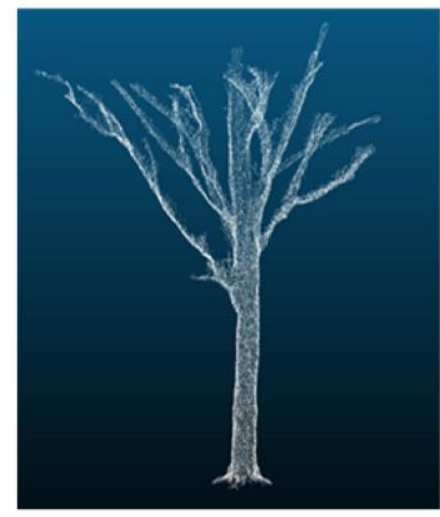

$\mathrm{c}$

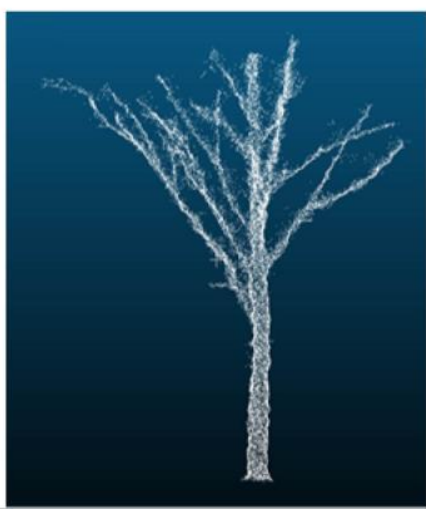

$f$

Note: (a) (b) (c) are in an indoor experimental environment; (d) (e) (f) are in an outdoor experimental environment; (a) (d) registration using ICP method; (b) (e)registration using NL_ICP method; (c) (f) registration using N_ICP method.

Figure 10 3D model of branches

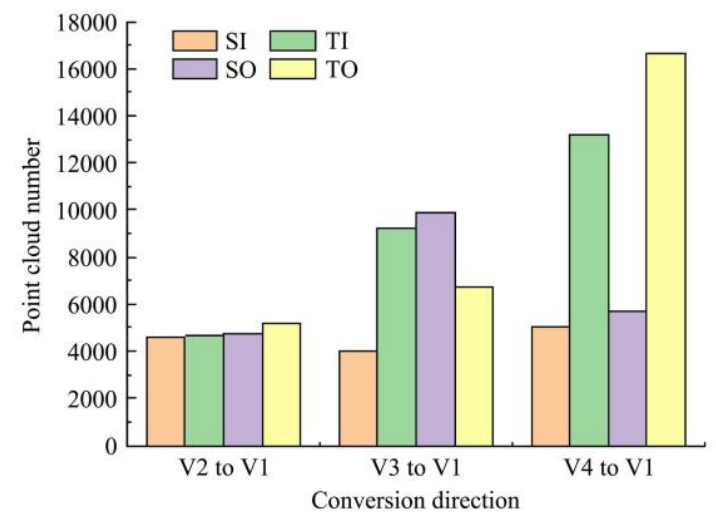

a. Branch point cloud number variation

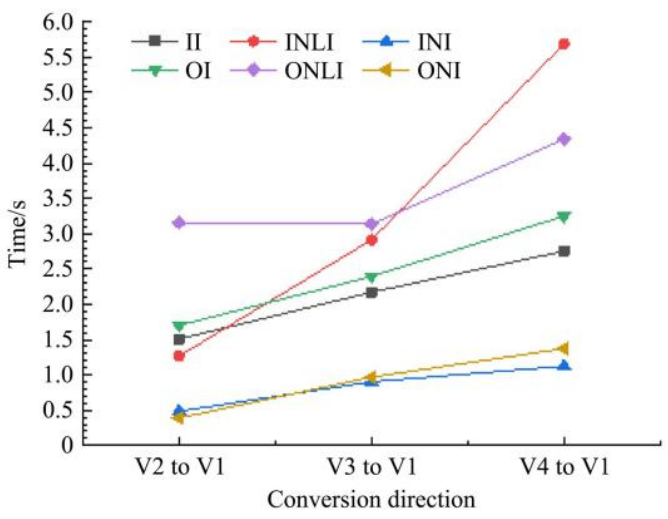

b. Running time of each step of various registration methods

Figure 11 Registration evaluation 
Table 3 Branch fitness score

\begin{tabular}{cccc}
\hline \multirow{2}{*}{$\begin{array}{c}\text { Algorithms in different } \\
\text { environments }\end{array}$} & \multicolumn{3}{c}{ Conversion direction } \\
\cline { 2 - 4 } & V2 to V1 & V3 to V1 & V4 to V1 \\
\hline II & 0.017419 & 0.001261 & 0.00201 \\
OI & 0.00222 & 0.000688 & 0.000153 \\
INLI & 0.01744 & 0.001226 & 0.001956 \\
ONLI & 0.002154 & 0.000705 & 0.000149 \\
INI & 0.018284 & 0.001631 & 0.002609 \\
ONI & 0.002194 & 0.000699 & 0.000143 \\
\hline
\end{tabular}

The registration results of the crown indoors and outdoors are shown in Figure 12. The number of point cloud changes in the registration process is shown in Figure 13a, the running time of the program is shown in Figure 13b, and the fitness score of crown registration is shown in Table 4. As shown in Figures 13a and $13 \mathrm{~b}$, the registration time of the point clouds increased with the increase of the number of point clouds in the point clouds in the registration process. In indoor scenes, for the crown, the minimum registration time can reach $3.056 \mathrm{~s}$. The corresponding registration method is N_ICP. The fitness score of registration is $0.0006179,0.0006175$ and 0.0006534 , respectively. The values are basically similar. In outdoor scenes, for the crown, the final registration time can reach $2.638 \mathrm{~s}$, the corresponding registration method is N_ICP. The fitness score is $0.0006563,0.0006601$ and 0.0006374 , respectively, with similar values. From the above experimental results, N_ICP can achieve the shortest registration time in both indoor and outdoor environments for the crown. ICP and its improved algorithm have no significant impact on the registration results.

Figures $9 \mathrm{~b}, 11 \mathrm{~b}$, and $13 \mathrm{~b}$ show that during the registration process, the NL_ICP registration method consumed longer than other registrations, both indoors and outdoors. Since the NL_ICP applied the Levenberg-Marquardt algorithm to optimize the registration results after registration, the run time will increase.

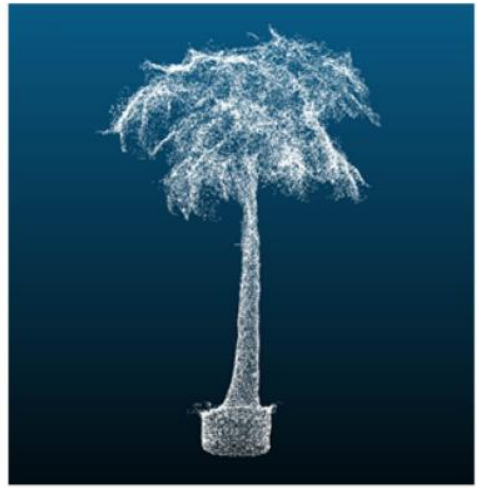

a

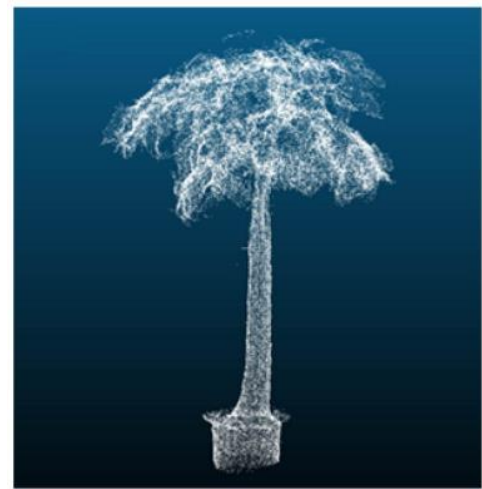

d

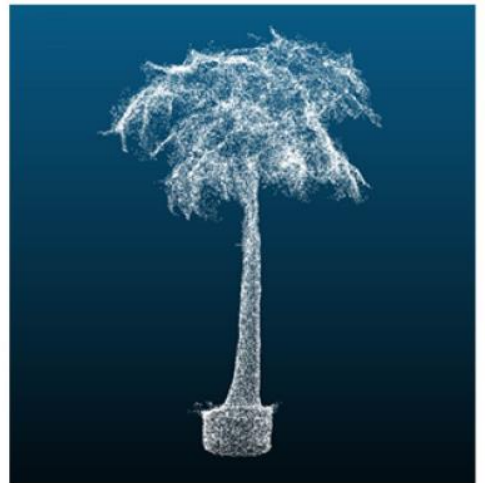

b

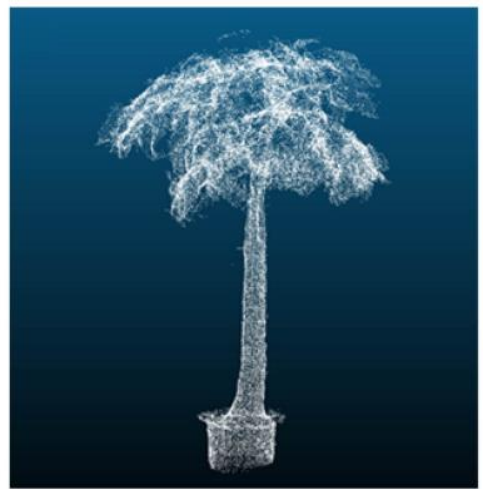

$\mathrm{e}$

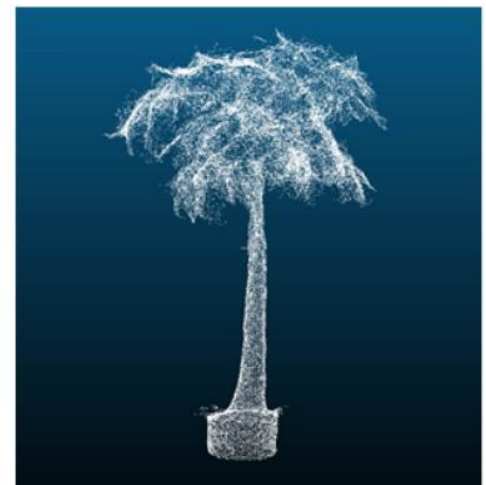

$\mathrm{c}$

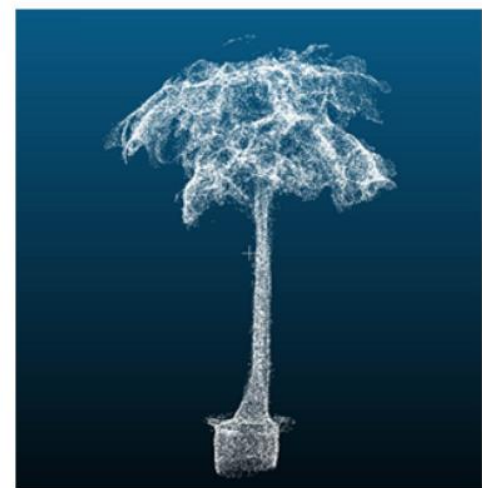

f

Note: (a) (b) (c) are in an indoor experimental environment; (d) (e) (f) are in an outdoor experimental environment; (a) (d) registration using ICP method; (b) (e) registration using NL_ICP method; (c) (f) registration using N_ICP method.

Figure 12 3D model of the crown

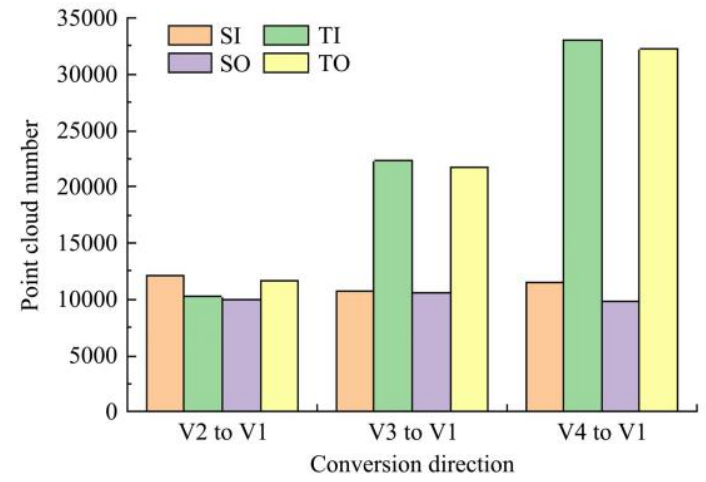

a. Crown point cloud number variation

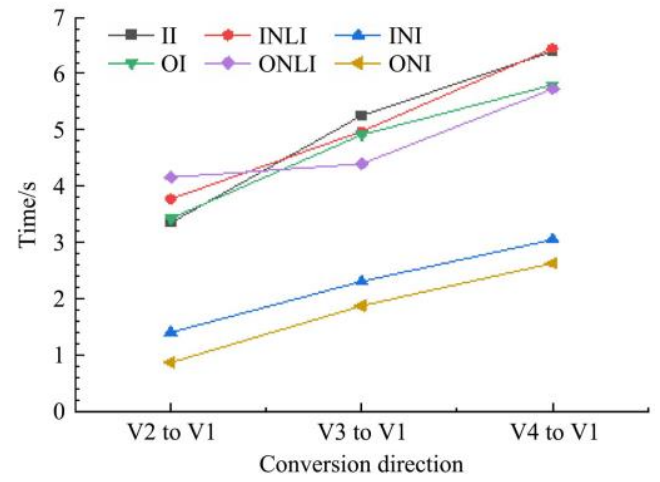

b. Running time of each step of various registration methods

Figure 13 Registration evaluation 
Table 4 Crown fitness score

\begin{tabular}{cccc}
\hline \multirow{2}{*}{$\begin{array}{c}\text { Algorithms in different } \\
\text { environments }\end{array}$} & \multicolumn{3}{c}{ Conversion direction } \\
\cline { 2 - 4 } & V2 to V1 & V3 to V1 & V4 to V1 \\
\hline II & 0.0022078 & 0.0012034 & 0.0006179 \\
OI & 0.0025886 & 0.0018664 & 0.0006563 \\
INLI & 0.0022133 & 0.0012077 & 0.0006175 \\
ONLI & 0.0025782 & 0.0018702 & 0.0006601 \\
INI & 0.0021832 & 0.0013533 & 0.0006534 \\
ONI & 0.0025796 & 0.0018675 & 0.0006374 \\
\hline
\end{tabular}

\section{Conclusions}

A point cloud registration method based on three calibration balls for 3D crop modelling was developed and validated in this research. According to this method, the interval of the viewpoints for collecting point cloud data could reach up to approximately $90^{\circ}$, which can solve the problems of low registration accuracy due to large viewing angle interval and elaborate data collection in the process of point cloud registration. Three objects such as trunk, branch, and crown were used for the registration in both indoor and outdoor environments. The results demonstrated that there was no significant difference in fitness score and running time in both indoor and outdoor conditions. The fitness score of ICP, NL_ICP and N_ICP ranged from 0.0000864 to 0.002609 , in which NL_ICP achieved the best registration accuracy (0.002609). Meanwhile, the running time of the three algorithms varied from 0.429 to $6.45 \mathrm{~s}$ and N_ICP consumed the shortest running time $(0.429 \mathrm{~s})$. For the same object whether indoor or outdoor, the proposed method performed steady robustness because there were no obvious differences in fitness score and running time using the three algorithms based on the calibration balls. It can be concluded that the developed method based on calibration balls was suitable for point cloud registration in both indoor and outdoor conditions, and can be considered as an effective means providing 3D point cloud data to crop modelling.

\section{Acknowledgements}

This research was funded by the National Key R\&D Program of China (Grant No. 2018YFD0700601) and the National Natural Science Foundation of China (Grant No. 31600588).

\section{[References]}

[1] Lin Y. LiDAR: An important tool for next-generation phenotyping technology of high potential for plant phenomics? Computers \& Electronics in Agriculture, 2015; 119: 61-73.

[2] Deans A R, Lewis S E, Huala E, Anzaldo S S, Ashburner M, Balhoff J P, et al. Finding Our Way through Phenotypes. Plos Biology, 2015; 13(1): e1002033.

[3] Karkee M, Adhikari B. A method for three-dimensional reconstruction of apple trees for automated pruning. Transactions of the ASABE, 2015; 58(3): 565-74.

[4] Colaco A F, Trevisan R G, Molin J P, Rosell-Polo J R, Escola A. A method to obtain orange crop geometry information using a mobile terrestrial laser scanner and 3D modeling. Remote Sensing, 2017; 9(8): 763.

[5] Li D W, Xu L H, Tang X S, Sun S Y, Cai X, Zhang P. 3D imaging of greenhouse plants with an inexpensive binocular stereo vision system. Remote Sensing, 2017; 9(5): 508.

[6] Li Y Y, Fan X C, Mitra N J, Chamovitz D, Cohen-Or D, Chen B Q. Analyzing growing plants from 4D point cloud data. ACM Transactions on Graphics, 2013; 32(6): 157.

[7] Li J, Tang L. Developing a low-cost 3D plant morphological traits characterization system. Computers and Electronics in Agriculture, 2017; 143: 1-13.

[8] Oliver A, Kang S, Wünsche B C, Macdonald B. Using the Kinect as a navigation sensor for mobile robotics. Conference on Image \& Vision Computing, New Zealand, 2012.

[9] Liao H J, Long X L. Study on virtual assembly system based on Kinect somatosensory interaction. International Conference on Information Science and Cloud Computing, Guangzhou, China, 2013.

[10] Hondori M H, Khademi M. A review on technical and clinical impact of Microsoft Kinect on physical therapy and rehabilitation. Journal of Medical Engineering, 2014; 2014(1): 846514.

[11] Boutsika E. Kinect in Education: A Proposal for children with autism. Procedia Computer Science, 2014; 27: 123-129.

[12] Vazquez-Arellano M, Reiser D, Paraforos D S, Garrido-Izard M, Burce M E C, Griepentrog H W. 3-D reconstruction of maize plants using a time-of-flight camera. Computers and Electronics in Agriculture, 2018; 145: 235-247.

[13] Gai J Y, Tang L, Steward B. Plant recognition through the fusion of 2D and 3D images for robotic weeding. ASABE Annual International Meeting, New Orleans, USA, Jul 26-29, 2015.

[14] Cheng X, Li Z W, Zhong K, Shi Y S. An automatic and robust point cloud registration framework based on view-invariant local feature descriptors and transformation consistency verification. Optics and Lasers in Engineering, 2017; 98: 37-45.

[15] Rusu R B, Cousins S. 3D is here: Point cloud library (PCL). IEEE International Conference on Robotics \& Automation, Shanghai, China; 2011.

[16] Chen H, Bhanu B. 3D free-form object recognition in range images using local surface patches. Pattern Recognition Letters, 2007; 28(10): 1252-1262.

[17] Zhang A L, Zhang B X, Song C J, Shen D P, Zhu E G, Dong F S Viewpoint calibration method based on point features for point cloud fusion. IEEE International Conference on Image Processing, Beijing, China; 2017.

[18] Kalman D. A singularly valuable decomposition: the SVD of a matrix The College Mathematics Journal, 1996; 27(1): 2-23.

[19] Lachat E, Macher H, Landes T, Grussenmeyer P. Assessment and calibration of a RGB-D camera (Kinect v2 sensor) towards a potential use for close-range 3D modeling. Remote Sensing, 2015; 7(10): 13070-13097.

[20] Yang L, Zhang L Y, Dong H W, Alelaiwi A, Saddik A E. Evaluating and improving the depth accuracy of Kinect for Windows v2. IEEE Sensors Journal, 2015; 15(8): 4275-4285.

[21] Miknis M, Davies R, Plassmann P, Ware A. Near real-time point cloud processing using the PCL. International Conference on Systems, Signals and Image Processing; 2015.

[22] Rusu R B, Blodow N, Marton Z C, Beetz M. Close-range scene segmentation and reconstruction of $3 \mathrm{D}$ point cloud maps for mobile manipulation in domestic environments. IEEE/RSJ International Conference on Intelligent Robots and Systems, St Louis, MO, USA, 2009.

[23] Hautamaki V, Karkkainen I, Franti P. Outlier detection using k-nearest neighbour graph. Proceedings of the 17th International Conference on Pattern Recognition, IEEE, 2004.

[24] Schnabel R, Wahl R, Klein R. Efficient RANSAC for point - cloud shape detection. Computer graphics forum; 2007: Wiley Online Library.

[25] Besl P J, McKay N D. Method for registration of 3-D shapes. In Proceedings of Sensor Fusion IV: Control paradigms and data structures, 1992; pp.586-607.

[26] Maiseli B, Gu Y F, Gao H J. Recent developments and trends in point set registration methods. Journal of Visual Communication and Image Representation, 2017; 46: 95-106.

[27] Alexa M, Behr J, Cohen-Or D, Fleishman S, Levin D, Silva C T. Computing and rendering point set surfaces. IEEE Transactions on Visualization and Computer Graphics, 2003; 9(1): 3-15.

[28] Xian Y R, Xiao J, Wang Y. A fast registration algorithm of rock point cloud based on spherical projection and feature extraction. Frontiers of Computer Science, 2019; 13: 170-182. 